\title{
Generating simple random graphs with prescribed degree distribution
}

\author{
Tom Britton * $\quad$ Maria Deijfen ${ }^{\dagger} \quad$ Anders Martin-Löf $\ddagger$
}

May 2006

\begin{abstract}
Let $F$ be a probability distribution with support on the non-negative integers. Four methods for generating a simple undirected graph with (approximate) degree distribution $F$ are described and compared. Two methods are based on the so called configuration model with modifications ensuring a simple graph, one method is an extension of the classical Erdős-Rényi graph where the edge probabilities are random variables, and the last method starts with a directed random graph which is then modified to a simple undirected graph. All methods are shown to give the correct distribution in the limit of large graph size, but under different assumptions on the degree distribution $F$ and also using different order of operations.
\end{abstract}

Keywords: Simple graphs, random graphs, degree distribution, generating algorithms, configuration model.

PACS: 02.50.-r; 89.75.Hc; 89.75.Da; 02.10.Ox

\section{Introduction}

A graph consists of two sets of objects: a set of vertices, $\mathcal{V}$, and a set of edges, $\mathcal{E}$. Each edge corresponds to a pair of vertices and the graph is said to be undirected if these pairs are unordered so that no directions are associated with the edges. Graphs with no duplicate edges and no loops - that is, with at most one edge between each pair of vertices and with no edges between a vertex and itself - are called simple. Furthermore, a graph is referred to as random if some kind of randomness is involved in its

*Department of Mathematics, Stockholm University.

$\dagger^{\dagger}$ Department of Mathematics, Stockholm University, 10691 Stockholm, Sweden, email: mia@math.su.se, fax: +4686126717.

${ }^{\ddagger}$ Department of Mathematics, Stockholm University. 
construction. In this paper we will consider graphs that are random in that the edges are generated by random mechanisms. The question at issue is, given a set of vertices and a probability distribution $F$ on the non-negative integers, how do we proceed to construct a simple undirected graph where the degree of a randomly chosen vertex has distribution $F$ ?

The simplest random graph model is the Erdös-Rényi graph, which was introduced in the late 50's by Paul Erdös and Alfréd Rényi [8, 9]. In fact they introduced two, closely related, models. Given a set of $n$ vertices and a number $m \leq\left(\begin{array}{l}n \\ 2\end{array}\right)$, the first model, denoted by $\mathcal{G}_{n, m}$, is defined as the ensemble of graphs having exactly $m$ edges, each possible graph appearing with equal probability. The second model, denoted by $\mathcal{G}_{n, p}$, is obtained by independently adding each one of the $\left(\begin{array}{c}n \\ 2\end{array}\right)$ possible edges of the graph with some probability $p$. The distribution of the vertex degree is then binomial with parameters $n-1$ and $p$ and, if $p$ is scaled by $1 / n$, we get a Poisson distribution in the limit as $n \rightarrow \infty$. Erdős-Rényi graphs have been widely studied and thorough descriptions of the field can be found in [4, 12].

An area that has received a lot of attention within statistical physics during the last few years is the use of graphs as models for various types of complex networks; see e.g. [7, 18, and the references therein. Examples of network structures that have been studied are social networks, power grids, the structure of the internet and various types of collaboration networks. Typically, this type of networks are very large, making it impossible to describe them in detail. A natural approach then is to let the edges representing the connections in the network be generated by a random procedure designed so that the resulting graph captures the features of the real-life network in question as well as possible. Since, as mentioned, the networks are usually large, it is particularly urgent that the asymptotic properties of the graph model agree with empirical observations.

An essential characteristic of a graph is the vertex degree and, in a random graph, this is a random quantity. For instance, as mentioned above, in the $\mathcal{G}_{n, p}$ model by Erdős and Rényi, the degree of a vertex is asymptotically Poisson distributed. The Erdős-Rényi graphs have a very simple and appealing mathematical structure and a lot of work has been done on the model. However, empirical studies have shown that the degree distribution in many real-life networks differs significantly from a Poisson distribution; see e.g. [15] (human sexual relationships), [10] (physical structure of the internet) and [1] (movie actor collaboration network). Complex networks typically have a more heavy-tailed degree distribution, often specified by some kind of power law, meaning that the number of vertices with degree $k$ is proportional to $k^{-\tau}$ for some exponent $\tau>1$. This type of graphs is often referred to as scale-free graphs and there are important features of 
such graphs that are missed out if they are approximated by Erdős-Rényi graphs; see e.g. [1].

In view of the above, it is important to be able to generate random graphs with other degree distributions than Poisson. The aim of this paper is to contribute at this point by describing a number of algorithms that, given a probability distribution $F$ (which will later be subject to various restrictions), produces simple undirected graphs whose vertex degree is asymptotically distributed according to $F$ (here, clearly it is required that $\operatorname{Supp}(F) \subseteq \mathbb{N}$, where $\operatorname{Supp}(F)$ denotes the support of $F$ ). To be more precise, given a set of $n$ vertices and a random mechanism to generate edges between them, let $p_{k}^{(n)}$ denote the probability of a randomly chosen vertex having degree $k$ and write $F=\left\{p_{k} ; k \geq 0\right\}$. Our task is then to design an edge mechanism such that

(i) $\lim _{n \rightarrow \infty} p_{k}^{(n)}=p_{k}$;

(ii) the resulting graph is simple and undirected.

In all applications mentioned above, the networks are simple and undirected. Other applications might involve more complex networks, for instance the link structure of the world-wide web constitutes a directed graph and bipartite graphs - that is, graphs with two types of vertices and edges running only between unlike types - are common within sociology. However, simple undirected networks is indeed an important class in applications. The present work provides a rigorous treatment of the asymptotic behavior of the vertex degree in a number of possible methods for generating such graphs. Some of the methods/results are new, but an important point of the paper is also to survey and gather previously known material. To our knowledge, this type of comparative study with focus on the vertex degree does not exist previously in the literature.

We mention also that there exist algorithms for generating simple graphs with a given degree sequence $d_{1}, \ldots, d_{n}$; here, $d_{i}$ is a non-negative integer specifying the degree of vertex $i$. For example, in McKay and Wormald [14] an algorithm is described which, under certain conditions, produces a uniformly selected simple undirected graph with the exact prescribed degree sequence. This is of course even better than the algorithms studied in the present paper which only has the correct degree distribution in the limit as the number of vertices tends to infinity. However, the price one has to pay for this is a more complicated algorithm and more restrictive assumptions about the degree distribution (the McKay and Wormald criterion corresponds to the degree distribution having moments of order $4+\epsilon)$. In the sequel we are hence not interested in obtaining a specific degree sequence, 
but only in proving that the distribution converges to the intended one in the limit.

The rest of the paper is organized as follows. In Section 2 we review the well-known configuration model and describe how it can be used to generate simple graphs with an arbitrary prescribed degree distribution. Section 3 treats a model inspired by Chung and Lu [5, 6] that generates simple graphs with mixed Poisson degree distributions. In Section 4 we propose a method that is based on the introduction of directed edges according to a suitably chosen distribution. This method produces graphs with a degree distribution whose generating function contains a Poisson factor. Finally, in Section 5 the methods are discussed and evaluated.

\section{The configuration model}

The configuration model was defined independently in 3 and 21, both papers being inspired by [2]. The model has later been analyzed in [16, 17, [19] for instance. Given a probability distribution $F$, the model describes a way to construct an undirected graph on $n$ vertices, labelled $v_{1}, \ldots, v_{n}$, having degree distribution $F$. It is defined as follows. For each vertex $v_{i}$, generate a degree $d_{i}$ independently from a random variable $D$ with distribution $F$ and attach $d_{i}$ "stubs" to $v_{i}$. Then join the stubs of all vertices pairwise completely at random to form edges between the vertices. To be more precise, first pick two stubs randomly among all stubs in the graph and join them. Then pick two stubs at random from the remaining $\sum_{1}^{n} d_{i}-2$ stubs and join them, etc.

A few problems might occur in the construction of a graph according to this algorithm. The first obvious problem is if the sum of all degrees, $\sum_{i} d_{i}$, happens to be an odd number. In this case there will always be one remaining stub left over in the pairing algorithm. However, unless $n$ is odd and $F$ is concentrated to the odd numbers, this problem is easily solved by either regenerating the degrees until their sum is even or removing one stub chosen at random. More serious problems arise when the aim is to generate a simple undirected graph, that is, a graph without loops and multiple edges. In the configuration model, it is clearly possible for a stub of a given vertex $v_{i}$ with $d_{i} \geq 2$ to be matched with another one of the stubs of $v_{i}$, resulting in an edge from vertex $v_{i}$ to itself, that is, a loop. Similarly, two stubs of $v_{i}$ could by chance be joined with two stubs of the same other vertex, with the effect that a multiple edge is created.

So what should we do if we insist on the resulting graph being simple? Two obvious suggestions are (1): to remove loops and merge multiple edges into single edges in the generated graph to obtain a simple graph as final 
product, or (2): to redo the algorithm until a simple graph occurs by chance. These methods will be referred to as "Erased configuration model" and "Repeated configuration model" respectively. Both methods make the degree distribution somewhat different from the intended one, but, as we will see, both of them have the right degree distribution asymptotically under certain moment conditions on the degree distribution.

\subsection{The erased configuration model}

Let $F_{n}=\left\{p_{j}^{(n)} ; j \geq 0\right\}$ denote the degree distribution in the erased configuration model with stub distribution $F=\left\{p_{j} ; j \geq 0\right\}$, that is, $p_{j}^{(n)}$ is the probability that a randomly selected vertex has degree $j$ in the erased configuration model on $n$ vertices. Also, write $N_{j}^{(n)}$ for the number of vertices having degree $j$ in the resulting graph.

Theorem 2.1 If $F$ has finite mean, then

(a) $F_{n} \rightarrow F$ as $n \rightarrow \infty$;

(b) $N_{j}^{(n)} / n \rightarrow p_{j}$ in probability, that is, the empirical distribution converges in probability to $F$.

The proof of this theorem is a bit lengthy, although not hard. It is found in the Appendix.

Theorem2.1 is not true without the assumption that the degree distribution $F$ has finite mean, at least not when the tail decays like $1-F(x) \sim c x^{-\alpha}$ where $\alpha<1$. A heuristic argument for this goes as follows. For such a decay of the tail, it is known that $\sum_{i=1}^{n} D_{i}$ and $\max _{\{1 \leq i \leq n\}} D_{i}$ are of the same order, see e.g. [11, Ch 13.11]. As a consequence, the probability that a stub is connected to a stub of the maximal node is non-negligible. It follows that any node with original degree 2 or more has positive probability (bounded away from 0) to have more than one stub connected to the maximal node. But whenever this happens, the degree of the node is decreased in the erased configuration model and it follows that the new degree distribution will converge to a distribution stochastically smaller than $F$.

In view of the above, there is no hope that the degree distribution will stay unaffected by the erasing procedure when the mean is infinite. However, if the degrees are conditioned to be smaller than $n^{a}$ for some $a \in(0,1)$ it turns out that Theorem 2.1 remains valid. Indeed, in many applications it is artificial to include vertices with degree larger than $n^{a}$ for some $a \in(0,1)$, and hence it is sometimes natural with this type of conditioned degrees. 
Write $F_{n, a}$ for the degree distribution in the erased configuration model on $n$ vertices where the number of stubs $D_{i}^{n, a}$ of vertex $v_{i}$ has distribution

$$
P\left(D_{i}^{n, a}=j\right)=\frac{P\left(D_{i}=j\right)}{P\left(D_{i} \leq n^{a}\right)} \quad \text { for } j=0, \ldots, n^{a},
$$

with $D_{i} \sim F$. Also, let $N_{j}^{(n, a)}$ be the number of vertices with degree $j$ in the graph after loops and multiple edges have been erased.

Theorem 2.2 For any $a \in(0,1)$, we have

(a) $F_{n, a} \rightarrow F$ as $n \rightarrow \infty$;

(b) $N_{j}^{(n, a)} / n \rightarrow p_{j}$ in probability.

Except for a few minor modifications, the proof of this theorem is analogous to the proof of Theorem 2.1. The modifications are described in the Appendix.

\subsection{The repeated configuration model}

The repeated configuration model consists of performing the configuration model until it produces a simple graph. As pointed out in 16, it follows from results in [13] that the probability of obtaining a simple graph in the configuration model converges to a strictly positive constant $c$ if the degree distribution has finite second moment. This implies that a simple graph is then obtained after a geometrically distributed number of tries. Of course, such a graph might not be typical for the configuration model. In particular one might suspect that the number of edges is somewhat smaller than normal, since there by chance were no multiple edges or loops. However, below we use the result from [16] to show that the resulting degree distribution converges to the right one provided that it has finite second moment. In fact, we show the stronger result that the empirical degree distribution converges to the intended distribution.

Let $F_{n}=\left\{p_{j}^{(n)} ; j \geq 0\right\}$ be the degree distribution of the repeated configuration model on $n$ vertices with stub distribution $F=\left\{p_{j} ; j \geq 0\right\}$ and write $N_{j}^{(n)}$ for the number of vertices having degree $j$ in the resulting graph.

Proposition 2.1 Assume that $F$ has finite second moment. Then

(a) $F_{n} \rightarrow F$ as $n \rightarrow \infty$;

(b) $N_{j}^{(n)} / n \rightarrow p_{j}$ in probability. 
Proof: We first show part (b). Let $D_{1}, \ldots, D_{n}$ be i.i.d. random variables with distribution $F$ and let $\tilde{p}_{j}^{(n)}=\left|\left\{D_{i} ; D_{i}=j, i=1, \ldots, n\right\}\right| / n$ denote the empirical distribution of these $n$ variables; here $|\cdot|$ denotes set cardinality. Also, write $S_{n}$ for the event that the configuration model on $n$ vertices produces a simple graph. The empirical distribution of the repeated configuration model is the same as the distribution of the vector with elements $\tilde{p}_{j}^{(n)}$ conditioned on $S_{n}$ and we hence have to show that

$$
P\left(\left|\tilde{p}_{j}^{(n)}-p_{j}\right|>\epsilon \mid S_{n}\right) \rightarrow 0 \text { as } n \rightarrow \infty \text { for any } \epsilon>0 \text { and any } j .
$$

Trivially, we have

$$
\begin{aligned}
P\left(\left|\tilde{p}_{j}^{(n)}-p_{j}\right|>\epsilon \mid S_{n}\right) & =\frac{P\left(\left|\tilde{p}_{j}^{(n)}-p_{j}\right|>\epsilon, S_{n}\right)}{P\left(S_{n}\right)} \\
& \leq \frac{P\left(\left|\tilde{p}_{j}^{(n)}-p_{j}\right|>\epsilon\right)}{P\left(S_{n}\right)} .
\end{aligned}
$$

The numerator here converges to 0 by the law of large numbers and, by the cited result of [16, the assumption that $F$ has finite second moment implies that $P\left(S_{n}\right) \rightarrow c>0$. Hence (1) follows.

To show (a), note that, since $0 \leq N_{j}^{(n)} / n \leq 1$, by dominated convergence, the result in (b) implies that $\mathrm{E}\left[N_{j}^{(n)} / n\right] \rightarrow p_{j}$. But $\mathrm{E}\left[N_{j}^{(n)}\right]=\sum_{i=1}^{n} p_{j}^{(n)}=$ $n p_{j}^{(n)}$, and the desired result follows.

\section{The generalized random graph}

In an Erdős-Rényi graph on $n$ vertices, the edges are defined by independent Bernoulli random variables $\left\{X_{i j}\right\}_{i<j}$ with $P\left(X_{i j}=1\right)=p$, the event $X_{i j}=$ 1 signifying the presence of an undirected edge between $v_{i}$ and $v_{j}$. By definition, $X_{j i}=X_{i j}$ for $i<j$ and $X_{i i}=0$ for all $i$. In this section, we consider a model where the probability $p_{i j}$ of an edge between two vertices $v_{i}$ and $v_{j}$ is allowed to depend on $i$ and $j$. Special cases of this have been considered in [5, 6, 20]. We will show that, if the probabilities $\left\{p_{i j}\right\}$ are picked randomly in a suitable way, we get a graph with a degree distribution that is easy to characterize in the limit when $n \rightarrow \infty$; see Theorem 3.1, Also, the degrees of the vertices are approximatively independent. The model will be referred to as the generalized random graph. 
First, we develop the model in more detail. To this end, let $X=\left\{X_{i j}\right\}_{i<j}$ be the array of edge indicators and write $P\left(X_{i j}=1\right)=p_{i j}=1-q_{i j}$. Since the indicators are independent, the probability density of $X$ is given by

$$
P(X=x)=\prod_{i<j} p_{i j}^{x_{i j}} q_{i j}^{1-x_{i j}} .
$$

Introducing the odds ratios $r_{i j}=p_{i j} / q_{i j}$ and noting that $p_{i j}=r_{i j} /\left(1+r_{i j}\right)$ and $q_{i j}=1 /\left(1+r_{i j}\right)$, this can be written

$$
P(X=x)=\prod_{i<j}\left(1+r_{i j}\right)^{-1} \prod_{i<j} r_{i j}^{x_{i j}} .
$$

Moreover, if we specialize to the situation where $r_{i j}=u_{i} u_{j}$ for some parameters $u=\left\{u_{i}\right\}_{i=1}^{n}$ with $u_{i} \geq 0$ and define $G(u):=\prod_{i<j}\left(1+u_{i} u_{j}\right)$, we get

$$
\begin{aligned}
P_{u}(X=x) & =G^{-1}(u) \prod_{i<j}\left(u_{i} u_{j}\right)^{x_{i j}} \\
& =G^{-1}(u) \prod_{i} u_{i}^{d_{i}(x)},
\end{aligned}
$$

where $d_{i}(x)$ is the degree of the vertex $v_{i}$ in the configuration $x$, that is, $d_{i}(x):=\sum_{j} x_{i j}$. This is a "canonical" distribution in the sense of statistical mechanics with sufficient statistics $\left\{d_{i}(X)\right\}$ and from (2) we see that the conditional distribution of $X$ given that $\left\{d_{i}(X)=d_{i}\right\}$ is uniform, that is, all graphs with a given degree sequence $\left\{d_{i}\right\}$ have the same probability. This is indeed a nice property of the model, motivating the use of the parametrization $r_{i j}=u_{i} u_{j}$ instead of the one defined by $p_{i j}=u_{i} u_{j}$ used in [5, 6].

To obtain a formula for the joint generating function of the degree vector $\left\{d_{i}(X)\right\}$, note that, by (2), we have

$$
\begin{aligned}
\mathrm{E}_{u}\left[\prod_{i} t_{i}^{d_{i}(X)}\right] & =\sum_{x} P_{u}(X=x) \prod_{i} t_{i}^{d_{i}(x)} \\
& =G^{-1}(u) \sum_{x} \prod_{i}\left(t_{i} u_{i}\right)^{d_{i}(x)} .
\end{aligned}
$$

Since $\sum_{x} P_{u}(X=x)=1$, it follows from (2) that $\sum_{x} \prod_{i} u_{i}^{d_{i}(x)}=G(u)$, and hence we get 


$$
\begin{aligned}
\mathrm{E}_{u}\left[\prod_{i} t_{i}^{d_{i}(X)}\right] & =G^{-1}(u) G(t u) \\
& =\prod_{i<j} \frac{1+t_{i} u_{i} t_{j} u_{j}}{1+u_{i} u_{j}} .
\end{aligned}
$$

Now consider the situation where the parameters $\left\{u_{i}\right\}$ are suitably scaled random variables, more precisely, we set $u_{i}=W_{i} / \sqrt{n}$, where $\left\{W_{i}\right\}$ are i.i.d. random variables with finite mean $\mu_{W}$ and $W_{i} \geq 0$. Write $\left\{D_{i}\right\}$ for the degrees of the vertices in this setting, that is, $D_{i}=d_{i}(X)=d_{i}(X(W))$. The following theorem specifies the liming distribution of the $D_{i}$ :s.

Theorem 3.1 Consider a generalized random graph on $n$ vertices with edge probabilities defined by $p_{i j} / q_{i j}=W_{i} W_{j} / n$, where $\left\{W_{i}\right\}$ are i.i.d. random variables with mean $\mu_{W}$ and finite moment of order $1+\varepsilon$ for some $\varepsilon>0$. We have:

(a) The limiting distribution of a degree variable $D_{k}$ as $n \rightarrow \infty$ is mixed Poisson with parameter $W_{k} \mu_{W}$.

(b) For any $m$, the variables $D_{1}, \ldots D_{m}$ are asymptotically independent.

Proof: By taking $t_{k}=t$, where $0 \leq t \leq 1$, and $t_{i}=1$ for $i \neq k$ in (3), it follows that

$$
\mathrm{E}\left[t^{D_{k}}\right]=\mathrm{E}\left[\prod_{i \neq k} \frac{1+W_{i} W_{k} t / n}{1+W_{i} W_{k} / n}\right]
$$

Using the Taylor expansion $\log (1+x)=x+O\left(x^{2}\right)$, we see that

$$
\prod_{i} \frac{1+W_{i} W_{k} t / n}{1+W_{i} W_{k} / n}=\exp \left\{\frac{W_{k} \sum_{i} W_{i}}{n}(t-1)+R_{n}\right\}
$$

where $R_{n}=O\left(W_{k}^{2} \sum_{i} W_{i}^{2} / n^{2}\right)$. To estimate $R_{n}$, note that $W_{i}^{2} \leq \max _{l}\left\{W_{l}\right\} W_{i}$. The law of large numbers implies that $\sum_{i} W_{i} / n \rightarrow \mu_{W}$ and, since the $W_{l}: \mathrm{s}$ have finite $1+\varepsilon$-moment, we have that $\max _{1 \leq l \leq n}\left\{W_{l}\right\} / n \rightarrow 0$. It follows that $R_{n}$ converges almost surely to 0 as $n \rightarrow \infty$. Hence

$$
\mathrm{E}\left[t^{D_{k}}\right] \rightarrow \mathrm{E}\left[e^{W_{k} \mu_{W}(t-1)}\right] \quad \text { as } n \rightarrow \infty,
$$

and part (a) follows. To establish (b), note that, by taking $t_{i}=1$ for $i>m$ in (31) and proceeding as in proving (a), it can be seen that

$$
\mathrm{E}\left[\prod_{i=1}^{m} t_{i}^{D_{i}}\right] \rightarrow \prod_{i=1}^{m} \mathrm{E}\left[e^{W_{i} \mu_{W}\left(t_{i}-1\right)}\right] \quad \text { as } n \rightarrow \infty .
$$


Hence the joint generating function of $\left(D_{1}, \ldots, D_{m}\right)$ asymptotically factorizes into a product of mixed Poisson generating functions, as desired.

Now recall that our task is to generate a simple random graph with a given degree distribution $F$. According to the above theorem, if $F$ is mixed Poisson with parameter distribution $Q$ with finite moment of order $1+\varepsilon$, then this can be done by using the generalized random graph model with i.i.d. weights $\left\{W_{i}\right\}$ distributed according to $Q / \sqrt{\mu_{Q}}$, where $\mu_{Q}$ denotes the mean of $Q$. As mentioned in the introduction, the degree distribution in many real-life networks is heavy-tailed, the probability of a vertex having degree $k$ being proportional to $k^{-\tau}$ for some exponent $\tau>1$. It is not hard to see that heavy-tailed mixed Poisson distributions with this type of power law behavior can be accomplished by choosing a heavy-tailed parameter distribution with the desired exponent.

In this context it is clearly of interest to know to what extent Theorem 3.1 is still true if the distribution of $\left\{W_{i}\right\}$ has a heavy tail such that $E\left[W_{i}\right]=$ $\infty$. Indeed, according to the theorem below, if we assume that the tail of the distribution varies regularly in the sense that $P\left(W_{i}>w\right) \sim c w^{-\alpha}$ as $w \rightarrow \infty$, for some $\alpha \in(0,1)$ and some constant $c>0$, then the distribution of the $D_{k}$ :s is still mixed Poisson, but with a different scaling and different mixing distribution. Note that a power law distribution with exponent $\tau \in(1,2)$ satisfies the tail condition with $\alpha=\tau-1$.

Theorem 3.2 Suppose that $\left\{W_{i}\right\}$ are i.i.d. with $P\left(W_{i}>w\right) \sim c w^{-\alpha}$, for some $\alpha \in(0,1)$ and $c>0$, and consider the generalized random graph with $p_{i j} / q_{i j}=W_{i} W_{j} / n^{1 / \alpha}$. Then:

(a) The limiting distribution of a degree variable $D_{k}$ is mixed Poisson with parameter $\gamma W_{k}^{\alpha}$ where $\gamma=c \int_{0}^{\infty}(1+x)^{-2} x^{-\alpha} d x$.

(b) For any $m$, the variables $D_{1}, \ldots, D_{m}$ are asymptotically independent.

Remark As pointed out above, the tail behavior of a mixed Poisson distribution with a power law parameter is determined by the parameter. Hence, for a mixed Poisson variable $Y$ with parameter $\gamma W_{k}^{\alpha}$, we have that

$$
\begin{aligned}
P(Y \geq y) & \approx P\left(W_{k}^{\alpha} \geq y\right) \\
& =P\left(W_{k} \geq y^{1 / \alpha}\right) \\
& \sim c y^{-1}
\end{aligned}
$$

that is, the distribution is a power law with exponent $\tau=2$. 
Proof of Theorem 3.2; As in Theorem 3.1] we have

$$
E\left[t^{D_{k}}\right]=E\left[\prod_{i \neq k} \frac{1+W_{i} W_{k} t / n^{1 / \alpha}}{1+W_{i} W_{k} / n^{1 / \alpha}}\right] .
$$

Let us first fix the value of $W_{k}$, say $W_{k}=w$. Then

$$
\Phi_{n}(w):=E\left[t^{D_{k}} \mid W_{k}=w\right]=E\left[\prod_{i \neq k} \varphi\left(\frac{W_{i}}{n^{1 / \alpha}}\right)\right],
$$

with $\varphi(x):=(1+x w t) /(1+x w)$. Write $V(x)$ for the distribution function of $W_{i}$. Since all $\left\{W_{i}\right\}$ are independent, we have

$$
\begin{aligned}
\Phi_{n}(w) & =\left(\int_{0}^{\infty} \varphi\left(\frac{x}{n^{1 / \alpha}}\right) V(d x)\right)^{n-1} \\
& =\left(1+\int_{0}^{\infty}(\varphi(x)-1) V\left(n^{1 / \alpha} d x\right)\right)^{n-1} \\
& =\left(1+\int_{0}^{\infty} \varphi^{\prime}(x)\left(1-V\left(n^{1 / \alpha} x\right)\right) d x\right)^{n-1}
\end{aligned}
$$

where the last equality follows from partial integration. In order to see that the last integral is $O(1 / n)$, note that

$$
n \int_{0}^{\infty} \varphi^{\prime}(x)\left(1-V\left(n^{1 / \alpha} x\right)\right) d x=\int_{0}^{\infty} \frac{\varphi^{\prime}(x)}{x^{\alpha}}\left(n^{1 / \alpha} x\right)^{\alpha}\left(1-V\left(n^{1 / \alpha} x\right)\right) d x .
$$

By the assumption, $y^{\alpha}(1-V(y))$ is a bounded function which converges to $c>0$ as $y \rightarrow \infty$, and hence, by bounded convergence,

$$
\begin{aligned}
\lim _{n \rightarrow \infty} \int_{0}^{\infty} \frac{\varphi^{\prime}(x)}{x^{\alpha}}\left(n^{1 / \alpha} x\right)^{\alpha}\left(1-V\left(n^{1 / \alpha} x\right)\right) d x & =c \int_{0}^{\infty} \frac{\varphi^{\prime}(x)}{x^{\alpha}} d x \\
& =(t-1) w^{\alpha} \gamma,
\end{aligned}
$$

where $\gamma:=c \int_{0}^{\infty}(1+x)^{-2} x^{-\alpha} d x$. It follows that $\lim _{n \rightarrow \infty} \Phi_{n}(w)=e^{(t-1) \gamma w^{\alpha}}$, which is recognized as the generating function of a Poisson distribution with mean $\gamma w^{\alpha}$. Integrating over $w=W_{k}$, we see that the limit distribution of $D_{k}$ is mixed Poisson with mean $\gamma W_{k}^{\alpha}$ and generating function

$$
E\left[t^{D_{k}}\right]=E\left[e^{(t-1) W_{k}^{\alpha}}\right]=\int_{0}^{\infty} e^{(t-1) w^{\alpha}} V(d x) .
$$


The proof of $(b)$ is analogous to the proof of Theorem 3.1 and is therefore omitted.

We finish this section by showing that the empirical degree distribution in the generalized random graph converges to the asymptotic mixed Poisson degree distribution in the graph. To this end, when $\left\{W_{i}\right\}$ have finite mean $\mu_{W}$, write $N_{k}^{(n)}$ for the number of vertices having degree $k$ in the generalized random graph with edge probabilities defined by $p_{i j} / q_{i j}=W_{i} W_{j} / n$, and let $F=\left\{p_{k} ; k \geq 0\right\}$ be a mixed Poisson distribution with parameter $W \mu_{W}$. Similarly, when $P\left(W_{i}>w\right) \sim c w^{-\alpha}$ for some $\alpha \in(0,1)$, the number of vertices having degree $k$ for edge probabilities defined by $p_{i j} / q_{i j}=W_{i} W_{j} / n^{1 / \alpha}$ is denoted by $N_{k}^{(n, \alpha)}$, and we write $F^{\alpha}=\left\{p_{k}^{\alpha} ; k \geq 0\right\}$ for a mixed Poisson distribution with parameter $\gamma W^{\alpha}$, where $\gamma$ is defined in Theorem 3.2

Proposition 3.1 As $n \rightarrow \infty$ in the generalized random graph, we have:

(a) If $\left\{W_{i}\right\}$ have finite moment of order $1+\varepsilon$, then $N_{k}^{(n)} / n \rightarrow p_{k}$ in probability for all $k$.

(b) If $P\left(W_{i}>w\right) \sim c w^{-\alpha}$ for some $\alpha \in(0,1)$, then $N_{k}^{(n, \alpha)} / n \rightarrow p_{k}^{\alpha}$ in probability for all $k$.

Proof: The proofs of part (a) and part (b) are analogous and we give here the proof of (a). Write $P^{(n)}$ for the probability law of the generalized random graph on $n$ vertices and let $\mathbf{1}_{\{\cdot\}}$ denote the indicator function. Clearly $N_{k}^{(n)}=\sum_{i=1}^{n} \mathbf{1}_{\left\{D_{i}=k\right\}}$ and hence, using symmetry, it follows that

$$
\begin{aligned}
\mathrm{E}\left[N_{k}^{(n)}\right] & =\sum_{i=1}^{n} P^{(n)}\left(D_{i}=k\right) \\
& =n P^{(n)}\left(D_{1}=k\right) .
\end{aligned}
$$

By Theorem 3.1 (a), we have $P^{(n)}\left(D_{1}=k\right) \rightarrow p_{k}$ as $n \rightarrow \infty$, meaning that $\mathrm{E}\left[N_{k}^{(n)} / n\right] \rightarrow p_{k}$. The desired result is now obtained from Chebyshev's inequality if we can show that $\operatorname{Var}\left(N_{k}^{(n)} / n\right) \rightarrow 0$. To do this, note that

$$
\begin{aligned}
\mathrm{E}\left[\left(N_{k}^{(n)}\right)^{2}\right] & =\mathrm{E}\left[\sum_{i} \mathbf{1}_{\left\{D_{i}=k\right\}}^{2}+\sum_{i \neq j} \mathbf{1}_{\left\{D_{i}=k\right\}} \mathbf{1}_{\left\{D_{j}=k\right\}}\right] \\
& =n P^{(n)}\left(D_{1}=k\right)+n(n-1) P^{(n)}\left(D_{1}=k, D_{2}=k\right),
\end{aligned}
$$

where the last equality follows from symmetry. By Theorem 3.1 (b), the variables $D_{1}$ and $D_{2}$ are asymptotically independent and hence we have 
$P^{(n)}\left(D_{1}=k, D_{2}=k\right) \rightarrow p_{k}^{2}$ as $n \rightarrow \infty$. Using the formula $\operatorname{Var}(X)=$ $\mathrm{E}\left[X^{2}\right]-\mathrm{E}[X]^{2}$, it follows that $\operatorname{Var}\left(N_{k}^{(n)} / n\right) \rightarrow 0$ as $n \rightarrow \infty$ and we are done.

\section{Directed graphs with removed directions}

In this section we propose a construction method where directed edges are introduced according to some distribution $G$. The directions of the edges are then disregarded and multiple edges are fused together, producing a graph whose asymptotic degree distribution $F$ is the convolution of the distribution $G$ and a Poisson distribution with parameter $\mu_{G}$, where $\mu_{G}$ denotes the mean of $G$; see Proposition 4.1.

To describe the construction, write $\mathcal{V}=\left\{v_{1}, \ldots, v_{n}\right\}$ for the vertex set. Let $G$ be a probability distribution with finite mean $\mu_{G}$ and $\operatorname{Supp}(G) \subset \mathbb{N}$, and write $\left\{g_{k}\right\}$ for the probabilities associated with $G$. Also, define $G_{n}$ via the probabilities

$$
g_{k}^{(n)}:= \begin{cases}g_{k} & \text { for } k=0,1, \ldots, n-2 \\ \sum_{k=n-1}^{\infty} g_{k} & \text { for } k=n-1 \\ 0 & \text { for } k \geq n\end{cases}
$$

that is, $G_{n}$ is a truncated version of $G$ with support on $\{0,1, \ldots, n-1\}$. The graph is constructed as follows:

1. Associate independently to each vertex $v_{i}$ a random variable $Y_{i}$ with distribution $G_{n}$, and add to the graph $Y_{i}$ directed edges pointing out from $v_{i}$. The vertices to be hit by the edges starting at $v_{i}$ are chosen randomly without replacement from $\mathcal{V} \backslash\left\{v_{i}\right\}$, independently for all vertices. This defines a directed random graph $\mathcal{G}_{\text {dir }}(n, G)=\left\{\mathcal{V}, \mathcal{E}_{\text {dir }}\right\}$.

2. To obtain a simple undirected graph $\mathcal{G}(n, G)=\{\mathcal{V}, \mathcal{E}\}$, the directions of the edges are disregarded and multiple edges are fused together, that is, an undirected edge between the vertices $v_{i}$ and $v_{j}$ is included in $\mathcal{E}$ as soon as at least one of the directed edges $\left(v_{i}, v_{j}\right)$ and $\left(v_{j}, v_{i}\right)$ is present in $\mathcal{E}_{\text {dir }}$.

Let $D_{i}$ denote the degree of the vertex $v_{i}$ in the resulting undirected graph. To find an expression for $D_{i}$, write $\mathcal{V}_{i}^{\text {out }}$ for the set of vertices that are hit by edges pointing out from $v_{i}$ in $\mathcal{E}_{d i r}$ and write $\mathcal{V}_{i}^{i n}$ for the set of vertices that sends outgoing edges to $v_{i}$ in $\mathcal{E}_{\text {dir }}$. Define $Z_{i}=\left|\mathcal{V}_{i}^{\text {in }} \cap \neg \mathcal{V}_{i}^{\text {out }}\right|$ (here, $\neg$ denotes set complement) so that, in words, $Z_{i}$ indicates the number of 
edges in $\mathcal{E}_{\text {dir }}$ pointing at $v_{i}$ and starting at vertices that are not hit by outgoing edges from $v_{i}$. Some thought reveals that

$$
D_{i}=Y_{i}+Z_{i}
$$

Clearly all variables $\left\{Z_{i}\right\}$ have the same distribution, which we denote by $H_{(n, G)}$. Also remember that $Y_{i} \sim G_{n}$ for all $i$. Hence the degree variables $\left\{D_{i}\right\}$ are identically distributed and we write $F_{(n, G)}$ for their distribution. The following theorem is the aforementioned characterization of the asymptotic degree distribution as the convolution of $G$ and a Poisson distribution with the same mean as $G$.

Theorem 4.1 As $n \rightarrow \infty$, we have

(a) $G_{n} \rightarrow G$;

(b) $H_{(n, G)} \rightarrow \operatorname{Po}\left(\mu_{G}\right)$, where $\operatorname{Po}\left(\mu_{G}\right)$ is a Poisson distribution with mean $\mu_{G}$;

(c) $F_{(n, G)} \rightarrow G * \operatorname{Po}\left(\mu_{G}\right)$.

Proof: The claim in (a) is immediate from the definition of $G_{n}$. To prove (b), fix a vertex $v_{k}$ and, for $i \neq k$, let $X_{i k}$ be a $0-1$ variable, indicating whether there is a directed edge from $v_{i}$ to $v_{k}$ in $\mathcal{E}_{d i r}$ or not. Since there are $Y_{i}$ outgoing edges from $v_{i}$ and the vertices to be hit by these edges are chosen randomly without replacement from the $n-1$ vertices in $\mathcal{V} \backslash\left\{v_{i}\right\}$, we have $P\left(X_{i k}=1\right)=Y_{i} /(n-1)$. Also, we have that

$$
Z_{k}=\sum_{i \neq k ; v_{i} \in \neg \mathcal{V}_{k}^{\text {out }}} X_{i k}
$$

Hence, by conditioning on $\left\{Y_{i}\right\}$, we obtain

$$
\mathrm{E}\left[t^{Z_{k}}\right]=\mathrm{E}\left[\prod_{i \neq k ; v_{i} \in \neg \mathcal{V}_{k}^{\text {out }}}\left(1+\frac{Y_{i}}{n-1}(t-1)\right)\right],
$$

and, since $\left\{Y_{i}\right\}$ are i.i.d. with distribution $G_{n}$ and $\left|\neg \mathcal{V}_{k}^{\text {out }} \backslash\left\{v_{k}\right\}\right|=n-1-Y_{k}$, it follows that

$$
\mathrm{E}\left[t^{Z_{k}}\right]=\left(1+\frac{\mu_{G_{n}}}{n-1}(t-1)\right)^{n-1} \mathrm{E}\left[\left(\frac{n-1}{n-1+\mu_{G_{n}}(t-1)}\right)^{Y_{k}}\right] .
$$

Here $\mu_{G_{n}}$ denotes the mean of the distribution $G_{n}$. As $n \rightarrow \infty$, the first factor on the right hand side converges to $e^{\mu_{G}(t-1)}$, which is recognized as 
the moment generating function of a Poisson variable with parameter $\mu_{G}$, and the left hand side converges to 1 . Hence part (b) is established.

To prove (c), note that $\mathrm{E}\left[t^{Y_{k}+Z_{k}}\right]=\mathrm{E}\left[t^{Y_{k}} \mathrm{E}\left[t^{Z_{k}} \mid Y_{k}\right]\right]$. The inner expectation is calculated as above and we get

$$
\mathrm{E}\left[t^{Y_{k}+Z_{k}}\right]=\left(1+\frac{\mu_{G_{n}}}{n-1}(t-1)\right)^{n-1} \mathrm{E}\left[\left(\frac{t(n-1)}{n-1+\mu_{G_{n}}(t-1)}\right)^{Y_{k}}\right]
$$

As $n \rightarrow \infty$, the second factor converges to the generating function of $G$ and, as pointed out above, the limit of the first factor is the moment generating function of a Poisson distribution with mean $\mu_{G}$.

Having proved that the asymptotic distribution of the vertex degree in $\mathcal{G}(n, G)$ is $G * \operatorname{Po}\left(\mu_{G}\right)$, the obvious question is which distributions can arise in this way. We will not give a full answer to this question - it is presumably difficult - but rather give a few examples of distributions that can indeed be obtained and also specify how the distribution $G$ of the number of outgoing arrows at the vertices should be chosen in these cases.

\section{Power law distribution}

First note that, if $G$ is a power law distribution with exponent $\tau$, then $G * \operatorname{Po}\left(\mu_{G}\right)$ will be so as well. Hence, if we are not interested in the exact form of the resulting degree distribution, but only in that its tail decays as a certain power law, then the $\mathcal{G}(n, G)$-model is clearly applicable.

\section{Poisson distribution}

The simplest case when the exact form of the resulting degree distribution is important is the Poisson distribution. Clearly, a Poisson distribution $F$ with parameter $\mu_{F}$ is accomplished by choosing $G$ to be a Poisson distribution with parameter $\mu_{F} / 2$.

\section{Mixed Poisson distribution}

A mixed Poisson distribution can be obtained as a limiting degree distribution in $\mathcal{G}(n, G)$ given a certain condition (5) on the law of the parameter. To see this, assume that $F$ is mixed Poisson with parameter law $Q$ with finite mean $\mu_{Q}$. The moment generating function of $F$ then equals

$$
\psi_{F}(t)=\int_{0}^{\infty} e^{x(t-1)} d Q(x)
$$

Since it should hold that $F=G * \operatorname{Po}\left(\mu_{G}\right)$, we have $\psi_{G}(t)=\psi_{F}(t) e^{-\mu_{G}(t-1)}$ and $\mu_{G}=\mu_{Q} / 2$, and hence

$$
\psi_{G}(t)=\int_{0}^{\infty} e^{\left(x-\mu_{Q} / 2\right)(t-1)} d Q(x)
$$


To ensure that this is the generating function of a probability distribution, let $\xi_{Q}=\inf \operatorname{Supp}(Q)$ and assume that

$$
\xi_{Q}-\mu_{Q} / 2>0
$$

Then

$$
\psi_{G}(t)=\int_{0}^{\infty} e^{y(t-1)} d \widetilde{Q}(y)
$$

where $\widetilde{Q}(y)=Q\left(y+\mu_{Q} / 2\right)$, that is, $\widetilde{Q}$ is the distribution $Q$ translated $\mu_{Q} / 2$ units to the left. This means that $G$ is mixed Poisson with parameter distribution $\widetilde{Q}$. Hence, a mixed Poisson distribution with parameter distribution $Q$ that satisfies $\xi_{Q}-\mu_{Q} / 2>0$, is obtained as a limiting degree distribution in $\mathcal{G}(n, G)$ by choosing $G$ to be mixed Poisson with parameter distribution $\widetilde{Q}$.

\section{Compound Poisson distribution}

Let $F$ be compound Poisson with Poisson parameter $\lambda$ and discrete summand distribution $R$ with finite mean $\mu_{R}$ and generating function $\psi_{R}(t)$ (recall that a compound Poisson distribution is the law of a sum of a Poisson number of i.i.d. random variables). Then $\mu_{F}=\lambda \mu_{R}$ and $\psi_{F}(t)=$ $e^{\lambda\left(\psi_{R}(t)-1\right)}$, and, if we want the limiting degree distribution in $\mathcal{G}(n, G)$ to be $F$, then we must have

$$
\begin{aligned}
\psi_{G}(t) & =\psi_{F}(t) e^{-\mu_{G}(t-1)} \\
& =e^{\lambda\left(\psi_{R}(t)-1\right)-\mu_{G}(t-1)} .
\end{aligned}
$$

Here, since $\mu_{G}=\lambda \mu_{R} / 2$ and $\psi_{R}(t)=\sum_{0}^{\infty} r_{k} t^{k}$, where $\left\{r_{k}\right\}$ denotes the probabilities associated with $R$, the exponent in (6) can be written as

$$
\lambda\left(\left(r_{0}+\frac{\mu_{R}}{2}\right)+\left(r_{1}-\frac{\mu_{R}}{2}\right) t+\sum_{k=2}^{\infty} r_{k} t^{k}-1\right) .
$$

Assume that

$$
r_{1}>\mu_{R} / 2,
$$

and introduce a new distribution $R^{\prime}$ by defining

$$
r_{k}^{\prime}= \begin{cases}r_{0}+\mu_{R} / 2 & \text { for } k=0 \\ r_{1}-\mu_{R} / 2 & \text { for } k=1 \\ r_{k} & \text { for } k \geq 2,\end{cases}
$$


that is, $R^{\prime}$ is obtained by transferring the mass $\mu_{R} / 2$ from the point 1 to the point 0 in the distribution $R$ (note that a consequence of (7) is that $R$ must be chosen so that $\mu_{R}<2$ ). With $R^{\prime}$ defined in this way we have

$$
\psi_{G}(t)=e^{\lambda\left(\psi_{R^{\prime}}(t)-1\right)},
$$

that is, $G$ is a compound Poisson distribution with Poisson parameter $\lambda$ and summand distribution $R^{\prime}$. Hence a compound Poisson distribution $F$ with summand distribution $R$ that satisfies (7) is obtained as limiting degree distribution in $\mathcal{G}(n, G)$ by choosing $G$ to be a compound Poisson distribution with summands distributed according to $R^{\prime}$.

\section{Concluding comments}

In the present paper, four different ways of generating simple undirected graphs with a prescribed degree distribution are described. The methods are referred to as the erased configuration model, the repeated configuration model, the generalized random graph and the directed graph with removed directions (DGRD) respectively. None of the methods is able to produce a graph that has the desired distribution exactly - that is, in a finite graph, a randomly selected vertex will not have exactly the correct degree distribution - but under certain regularity assumptions, it is shown that all four methods give the right distribution in the limit as the number of vertices $n$ tends to infinity.

Let us summarize the assumptions on the degree distribution for the different methods: In order for the repeated configuration model to produce a simple graph in stochastically bounded time as $n \rightarrow \infty$, the second moment of the degree distribution has to be finite and for the generalized random graph model with edge probabilities scaled by $n$ to be applicable, finite moment of order $1+\varepsilon$ for some $\varepsilon>0$ is required. For the other two methods, finite mean is sufficient. If the degrees are conditioned on being smaller than $n^{a}$ for some $a \in(0,1)$, the erased configuration model can handle distributions with infinite mean as well, and, with a different scaling of the edge probability, also the generalized random graph can be applied to infinite mean distributions.

As for the class of achievable distributions, the erased configuration model and the repeated configuration model are both able to generate graphs with any limiting distribution. The generalized random graph model can only produce mixed Poisson distributions and the DGRD-model gives distributions that can be expressed as the convolution of a discrete distribution with finite mean and a Poisson distribution with the same mean - a class containing certain types of mixed Poisson and compound Poisson distributions 
for instance. However, if only tail properties of the desired distribution are specified, both the generalized random graph model and the DGRD-model can do the job.

Concerning the number of operations needed to produce the graph, it is easily seen to be of order $n$ for all methods except the generalized random graph, which requires $O\left(n^{2}\right)$ operations. Note however that an approximation of the generalized random graph model that uses only $O(n)$ operations can be obtained by replacing the conditional degree distribution of a given vertex, conditional on the $W_{i}$ :s, with a Poisson distribution with the same mean. Among the methods using $O(n)$ operations the erased configuration model and the DGRD-model require less operations: The repeated configuration model generates a graph a geometrically distributed number of times whereas the other two methods only generates a graph once and then erases a few edges.

A perhaps more subjective opinion is that the generalized random graph model is probabilistically more tractable than the other methods. Its construction is straightforward, containing less dependence structures, implying that it is easier to show property results for this model. Also, it was easily seen from the construction of the general random graph that the obtained graph is uniform in the sense that all graphs with a given degree sequence have the same probability.

Finally we mention that, apart from the degree distribution, there is of course a number of other properties of a graph of which it would also be desirable to have control over, for instance clustering and path length. Rigorous analyzes of algorithms incorporating these aspects is to a large extent lacking and it is a future task to further investigate and develop methods for generating random graphs specifying such properties.

Acknowledgement We thank Remco van der Hofstad for giving the idea of how to strengthen Theorem 2.1 to cover also the empirical degree distribution, and for suggesting Theorem 2.2. Maria Deijfen also thanks van der Hofstad for interesting and enlightening discussions on the topic of random graphs.

\section{References}

[1] Barabási, A.-L. and Albert, R. (1999): Emergence of scaling in random networks, Science 286, 509-512.

[2] Bender, E. and Canfield, E. (1978): The asymptotic number of labelled graphs with given degree sequences, J. Comb. Th. A 24, 296-307. 
[3] Bollobás, B. (1980): A probabilistic proof of an asymptotic formula for the number of labelled regular graphs. European J. Combin., 1, 311-316.

[4] Bollobás, B. (2001): Random Graphs, Academic Press, 2nd ed.

[5] Chung, F. and Lu, L. (2002:1): Connected components in random graphs with given degrees sequences, Ann. Comb. 6, 125-145.

[6] Chung, F. and Lu, L. (2002:2): The average distances in random graphs with given expected degrees, Proc. Natl. Acad. Sci. 99, 15879-15882.

[7] Dorogovtsev, S. and Mendes, J. (2003): Evolution of Networks, from Biological Nets to the Internet and $W W W$, Oxford University Press.

[8] Erdős, P. and Rényi, A. (1959): On random graphs, Publ. Math. 6, 290-297.

[9] Erdős, P. and Rényi, A. (1960): On the evolution of random graphs, Publ. Math. Inst. Hung. Acad. Sci. 5, 17-61.

[10] Faloutsos, M., Faloutsos P. and Faloutsos, C. (1999): On power-law relationships of the internet topology, Comp. Comm. Rev. 29, 251-262.

[11] Feller, W (1966): An introduction to probability theory and its application, vol II, John Wiley.

[12] Janson, S., Luczak, T. and Rucinski, A. (1999): Random Graphs, John Wiley.

[13] McKay, B. (1985): Asymptotics for symmetric 0-1 matrices with prescribed row sums, Ars. Comb. 19A, 15-20.

[14] McKay, B.D. and Wormald, N.C.(1990): Uniform generation of random regular graphs of moderate degree, J. Algorithms 11, 52-67.

[15] Liljeros, F., Edling, C., Amaral, L., Stanley, H. and Åberg, Y. (2001): The web of human sexual contacts, Nature 411, 907-908.

[16] Molloy, M. and Reed, B. (1995): A critical point for random graphs with a given degree sequence, Rand. Struct. Alg. 6, 161-179.

[17] Molloy, M. and Reed, B. (1998): The size of the giant component of a random graphs with a given degree sequence, Comb. Prob. Comp. 7, 295305.

[18] Newman (2003): The structure and function of complex networks, SIAM Rev. 45, 167-256.

[19] Newman, M., Strogatz, S. and Watts, D. (2001): Random graphs with arbitrary degree distributions and their applications, Phys. Rev. E 64, 026118.

[20] Söderberg, B. (2002): A general formalism for inhomogeneous random graphs. Phys. Rev. E 66, 066121.

[21] Wormald, N.C. (1978): Some problems in the enumeration of labelled graphs, Doctoral thesis, Newcastle University. 


\section{Appendix}

Proof of Theorem 2.1; First note that, since $0 \leq N_{j}^{(n)} / n \leq 1$, by dominated convergence, the result in (b) implies that $\mathrm{E}\left[N_{j}^{(n)} / n\right] \rightarrow p_{j}$. But $\mathrm{E}\left[N_{j}^{(n)}\right]=\sum_{i=1}^{n} p_{j}^{(n)}=n p_{j}^{(n)}$, and hence (a) follows from (b).

To show (b), write $\widetilde{N}_{j}^{(n)}$ for the number of vertices that has degree $j$ before edges are erased to make the graph simple. By the law of large numbers $\widetilde{N}_{j}^{(n)} / n \rightarrow p_{j}$ as $n \rightarrow \infty$ and hence we are done if we can show that $\left(\widetilde{N}_{j}^{(n)}-N_{j}^{(n)}\right) / n \rightarrow 0$ in probability as $n \rightarrow \infty$. Let $M^{(n)}$ be the number of vertices where at least one stub is removed in the erasing procedure and note that $\widetilde{N}_{j}^{(n)}-N_{j}^{(n)} \leq M^{(n)}$. Hence, by Markovs inequality, it suffices to show that $\mathrm{E}\left[M^{(n)}\right] / n \rightarrow 0$. To do this, for $i=1, \ldots, n$, let $E_{i}$ be the number of stubs attached to $v_{i}$ that are rubbed out in the erasing procedure and define

$$
M_{i}^{(n)}= \begin{cases}1 & \text { if } E_{i} \geq 1 \\ 0 & \text { if } E_{i}=0 .\end{cases}
$$

Also, let $D_{i}$ be the degree of vertex $v_{i}$ before loops and multiple edges have been erased and write $P^{(n)}$ for the probability law of the erased configuration model on $n$ vertices (that is, averaged out also over the original degrees $\left\{D_{i}\right\}$ ). Since $M^{(n)}=\sum_{i=1}^{n} M_{i}^{(n)}$ and $\left\{M_{i}^{(n)}\right\}$ are equally distributed, we have

$$
\begin{aligned}
\frac{1}{n} \mathrm{E}\left[M^{(n)}\right] & =\frac{1}{n} \sum_{i=1}^{n} \mathrm{E}\left[M_{i}^{(n)}\right] \\
& =\mathrm{E}\left[M_{1}^{(n)}\right] \\
& =P^{(n)}\left(E_{1} \geq 1\right)
\end{aligned}
$$

The work lies in proving that $P^{(n)}\left(E_{1} \geq 1\right) \rightarrow 0$ as $n \rightarrow \infty$, or equivalently, that $P^{(n)}\left(E_{1}=0\right) \rightarrow 1$. Clearly this follows if we show that

$$
P^{(n)}\left(E_{1}=0 \mid D_{1}=j\right) \rightarrow 1 \text { for all } j \text { as } n \rightarrow \infty .
$$

To prove (8), write $A_{j}$ for the event that a given stub belonging to a vertex with $j$ stubs in total avoids being removed in the erasing procedure. Below we show that

$$
P^{(n)}\left(A_{j}\right) \rightarrow 1 \text { for all } j \text { as } n \rightarrow \infty .
$$

This establishes (8): After having merged one of the $j$ stubs of the vertex $v_{1}$ to a stub belonging to some other vertex, saving it from being 
erased, the probability that a fixed one of the other $j-1$ stubs are erased equals $P^{(n)}\left(A_{j-1}\right)$, since the fact that one stub from the other vertices is no longer available for merging is asymptotically negligible. This can then be repeated until there are no remaining stubs of $v_{1}$ and (8) follows by noting that $P^{(n)}\left(E_{1}=0 \mid D_{1}=0\right)=1$.

For the proof to be complete it remains to prove (9). To do this, first remember that a stub can be erased for two reasons: because it forms a loop and because it is part of a multiple edge. For the sake of completeness we also include the case when a randomly selected stub is removed if the total number of stubs is odd. Now, consider a fixed stub belonging to a vertex $v$ with $j$ stubs in all. Write $A_{j}^{l o o p}$ and $A_{j}^{\text {mult }}$ for the events that the stub is not part of a loop and a multiple edge respectively and let $A_{j}^{\text {odd }}$ be the event that the stub is not removed as the randomly selected "odd" stub. To estimate the probabilities of these events, we condition on that the total number of stubs equals $m$ and write $P_{m}^{(n)}$ for the corresponding conditioned probability measure. If $m$ is odd, the probability that the stub is removed as the "odd" stub is $1 / m$ and, if $m$ is even, the probability is 0 . Hence

$$
P_{m}^{(n)}\left(A_{j}^{o d d}\right) \geq 1-\frac{1}{m}
$$

For the stub to form a loop it has to be joined to one of the other $j-1$ stubs of the vertex $v$ and, since clearly the stub is matched to each one of the other $m-1$ stubs in the graph with the same probability $1 /(m-1)$, this happens with probability $(j-1) /(m-1)$, that is,

$$
P_{m}^{(n)}\left(A_{j}^{l o o p}\right)=1-\frac{j-1}{m-1} .
$$

To compute the probability that the stub is not part of a multiple edge, assume that it does not make up a loop and condition on the degree $k$ of the vertex $v^{\prime}$ of the stub to which it is joined. Also, number the remaining $j-1$ stubs of the vertex $v$ in some arbitrary way from 1 to $j-1$ and let $B_{j}$ be the event that there are no loops among these stubs. Trivially,

$$
P^{(n)}\left(A_{j}^{\text {mult }}\right) \geq P^{(n)}\left(A_{j}^{\text {mult }} \cap B_{j}\right) .
$$

For the event $A_{j}^{\text {mult }} \cap B_{j}$ to happen, none of the remaining $j-1$ stubs of the vertex $v$ can connect to another stub of $v$ or to a stub originating from $v^{\prime}$. Considering the stubs $1, \ldots, j-1$ one in a turn, we see that the probability that stub number 1 avoids being connected to a stub of $v$ or $v^{\prime}$ is $(m-j-k) /(m-3)$ (the denominator comes from that two stubs are already used in the fixed connection between $v$ and $v^{\prime}$ and the stub cannot join to itself). Then, given that stub number 1 is connected to some other vertex, 
the probability that stub number 2 is so as well is $(m-j-k-1) /(m-5)$, and so on. Furthermore, it is only possible for $A_{j}^{\text {mult }} \cap B_{j}$ to happen if $j-1 \leq m-k-j$, since otherwise loops among the $j-1$ stubs on vertex $v$ or multiple edges between $v$ and $v^{\prime}$ cannot be avoided. Hence, writing $P_{m, k}^{(n)}$ for the probability measure conditioned on $m$ and $k$, we have

$P_{m, k}^{(n)}\left(A_{j}^{m u l t} \cap B_{j}\right)= \begin{cases}\frac{m-k-j}{m-3} \frac{m-k-j-1}{m-5} \cdots \frac{m-k-2 j+2}{m-(2 j-1)} & \text { if } j-1 \leq m-k-j ; \\ 0 & \text { otherwise, }\end{cases}$

implying that

$$
P_{m, k}^{(n)}\left(A_{j}^{m u l t}\right) \geq\left(\frac{m-k-2 j+2}{m-3}\right)_{+}^{j-1},
$$

where $r_{+}=\max \{r, 0\}$. Combining (10), (11) and (12) and using Boole's inequality, it follows that

$$
\begin{aligned}
P_{m, k}^{(n)}\left(A_{j}\right) & =P_{m, k}^{(n)}\left(A_{j}^{\text {odd }} \cap A_{j}^{\text {loop }} \cap A_{j}^{\text {mult }}\right) \\
& \geq\left(\frac{m-k-2 j}{m}\right)_{+}^{j-1}-\frac{1}{m}-\frac{j-1}{m-1} .
\end{aligned}
$$

Removing the conditioning on $m$ and $k$ and denoting the corresponding random variable $L_{n}$ and $K_{n}$ respectively, we get

$$
P^{(n)}\left(A_{j}\right) \geq \mathrm{E}\left[\left(\frac{L_{n}-K_{n}-2 j}{L_{n}}\right)_{+}^{j-1}\right]-\mathrm{E}\left[\frac{1}{L_{n}}\right]-\mathrm{E}\left[\frac{j-1}{L_{n}-1}\right] .
$$

To complete the proof, we use dominated convergence to show that the right hand side of (13) converges to 1 as $n \rightarrow \infty$, establishing (9). Recall that $L_{n}$ is the total number of stubs in the configuration and $K_{n}$ is the number of stubs connected to the vertex of a randomly selected stub. The total number of stubs is a sum of $n$ i.i.d. random variables $\left\{D_{l}\right\}$ with distribution $F$ and mean $\mu_{F}$, which is finite by assumption. Hence, the law of large numbers implies that $L_{n} / n \rightarrow \mu_{F}$ almost surely as $n \rightarrow \infty$. The conditional distribution of $K_{n}$ given $\left\{N_{i}^{(n)}\right\}$ is specified by $P\left(K_{n}=\right.$ $i)=i N_{i}^{(n)} / \sum_{r} r N_{r}^{(n)}$, where $N_{i}^{(n)}$ is the number of $D_{l}$ :s that equal $i$. Since $N_{i}^{(n)} / n \rightarrow p_{i}$ as $n \rightarrow \infty$ and $\mu_{F}<\infty$, it follows that $K_{n}$ converges in distribution to a proper random variable $K$ with distribution $P(K=$ $i)=i p_{i} / \sum_{r} r p_{r}=i p_{i} / \mu_{F}$ and hence $K_{n} / n \rightarrow 0$ almost surely as $n \rightarrow \infty$. Combining these two observations, we get that

$$
\left(\frac{L_{n}-K_{n}-2 j}{L_{n}}\right)_{+}^{j-1} \rightarrow 1 \text { a.s. as } n \rightarrow \infty \text {. }
$$


Furthermore, since $j$ and $K_{n}$ are both strictly positive, we have

$$
0 \leq\left(\frac{L_{n}-K_{n}-2 j}{L_{n}}\right)_{+}^{j-1} \leq 1 .
$$

By dominated convergence, it follows that the first term on the right hand side of (13) converges to 1 , and it is easily seen that the other two terms converge to 0 . Hence the proof is complete.

Proof of Theorem 2.2, Fix $a \in(0,1)$ and let the degrees be distributed according to $F_{n, a}$. Clearly, if $F$ has finite mean, the claim follows immediately from Theorem 2.1, so assume that $F$ has infinite mean. The proof of Theorem 2.1 then remains valid all the way to the estimate (13). We need to see that the right hand side tends to 1 for all $j$ as $n \rightarrow \infty$. To this end, let $\tilde{\mu}$ be the expectation of $F$ conditioned on being smaller than, say, the smallest possible value in the support plus 10 . Then clearly $P\left(L_{n}>\tilde{\mu} n\right) \rightarrow 1$ as $n \rightarrow \infty$, and, trivially,

$\mathrm{E}\left[\left(\frac{L_{n}-K_{n}-2 j}{L_{n}}\right)_{+}^{j-1}\right] \geq \mathrm{E}\left[\left(\frac{L_{n}-K_{n}-2 j}{L_{n}}\right)_{+}^{j-1} \mid L_{n}>\tilde{\mu} n\right] P\left(L_{n}>\tilde{\mu} n\right)$.

On the event $\left\{L_{n}>\tilde{\mu} n\right\}$, we have $K_{n} / L_{n} \leq n^{a} / \tilde{\mu} n \rightarrow 0$. Hence, by bounded convergence, the right hand side above tends to 1 as $n \rightarrow \infty$ and, since the other two terms in the estimate (13) clearly converge to 0 , we are done. 